\title{
Imago Mundi: un acercamiento al internacionalismo intelectual $^{1}$
}

\section{Imago Mundi: an approach to intellectual internationalism}

\author{
Mariana Fernández Talavera \\ Universidad Nacional de Tres de Febrero \\ marianaft85@gmail.com
}

\begin{abstract}
En las páginas de la revista Imago Mundi (1953-1956) publicada en Buenos Aires, nos encontramos con una gran cantidad de artículos que se refieren a conocimiento que está produciéndose fuera de la Argentina. En este trabajo, vinculamos esta característica a cierto diagnóstico de poca y pobre producción intelectual nacional durante los años del gobierno peronista. En este sentido, analizamos aquí los vínculos o redes que se dan a través de la revista con lo que está produciendo en Europa y cómo esto configura el ideal cultural al que esta revista aspira así como también una representación de la labor intelectual durante esos años.
\end{abstract}

\section{Palabras clave}

Revistas, intelectuales, internacionalismo, universidad, peronismo

\begin{abstract}
In the pages of the journal Imago Mundi (1953-1956) published in Buenos Aires, we can find a big amount of articles in reference to the knwoledge that it is produced outside from Argentina. In this work, we try to connect this aspect of the journal with the diagnosis of a scanty intellectual national production during the years of the peronist goverment. As a result we analize the connections between the journal and what it is producing in Europe and how this configured the cultural ideal of this journal and the representation of the intellectual role in those years.
\end{abstract}

\section{Key words}

Journals, intellectuals, internacionalism, university, peronism

\footnotetext{
${ }^{1}$ Agradezco a los comentadores de la primera versión de este trabajo ya que gracias a sus aportes pude revisar y profundizar algunos aspectos que enriquecieron la investigación.
}

Esta obra está sujeta a la Licencia Reconocimiento-NoComercial-CompartirIgual 4.0 Internacional de Creative Commons. http://creativecommons.org/licenses/by-nc-sa/4.0/ 


\section{Mariana Fernández Talavera}

\section{Introducción}

El interés por la revista Imago Mundi, surge algunos años atrás cuando me encontraba leyendo la revista Centro, revista realizada por el Centro de Estudiantes de la Facultad de Filosofía y Letras de la Universidad de Buenos Aires en la década de 1950. En esta publicación había una sección dedicada a reseñar revistas de la época y fue allí, en esa recensión, que leí lo siguiente: "Lo que más interesa destacar [de Imago Mundi] es que el acto de darle vida quiere ser afirmativo, aun a costa de todas las dificultades"2. En este caso, se refiere justamente al objeto de estudio de Imago Mundi, que se plantea como una revista de "historia de la cultura". Lo que ven aquí los jóvenes intelectuales de Centro es un gesto por sí destacable: dedicar tiempo y esfuerzo a hacer una revista aun cuando no parezca haber destinatarios para ella. Esto se debe a que, para quienes participan de Centro, los años de gobierno peronista son años de abulia intelectual y no ven que haya incentivo para la producción cultural. Es por ello que rescatan el gesto de quienes hacen Imago Mundi como un modo de rescatar también su propio trabajo. De hecho, muchos de los que participaron en Imago Mundi, eran docentes que habían perdido su lugar en la universidad por cuestiones político-ideológicas. No obstante, los estudiantes nucleados en el Centro de Estudiantes de la Facultad seguirán considerándolos como referentes. Esto plantea un vínculo entre aquellos que están dentro de la universidad y aquellos que no se encuentran trabajando en este espacio. Ese fue el puntapié inicial para acercarme a las páginas de Imago Mundi.

Una vez que uno comienza a leer la revista nota inmediatamente la poca cantidad de referencias a la realidad nacional. En la revista predominan los artículos sobre autores extranjeros, al igual que las reseñas de congresos y de libros de autores extranjeros. Recordé entonces el diagnóstico hecho por Centro: poca o casi nula producción intelectual nacional. Y me pareció que la relación entre ambas publicaciones podía permitirnos pensar respecto de la actividad intelectual de esos años.

Es por ello que en el presente trabajo nos proponemos examinar las páginas de la revista Imago Mundi con el fin de analizar allí la mirada hacia el exterior y en función de ello ver qué idea de intelectual se está pensando desde esta revista, prestando especial atención a la presencia de lo internacional en sus páginas. Para esto será importante analizar los vínculos entre intelectuales a través de la noción de "redes intelectuales" de Devés Valdés. Es importante tener en cuenta también algunos rasgos de la universidad en esos años y los espacios alternativos a ella que surgieron como modo de producción intelectual. Lo que nos proponemos en estas páginas es analizar el contenido de la revista Imago Mundi y a partir de allí ver con qué tipo de intelectualidad se propugna de ese modo.

${ }^{2}$ Rodolfo Borello. "Imago Mundi”. Centro. Revista del centro de estudiantes de la facultad de Filosofía y Letras, número 7, año 3, Buenos Aires, diciembre de 1953, pág. 52. 


\section{La universidad, las revistas y las redes intelectuales}

Imago Mundi se publica entre 1953 y 1955, años de conflicto político en nuestro país, conflicto que no permanece ajeno a los sectores intelectuales. De hecho el nivel de conflictividad entre el gobierno y los intelectuales es bastante alto. Esto en parte tiene que ver con la intervención de la Universidad de Buenos Aires en 1946 que se extendió hasta 1950³. Esta intervención implicó cesantías de docentes, jubilaciones anticipadas y cierre de locales de algunos centros de estudiantes. Se implementó una nueva ley universitaria -la ley 13031- que no contemplaba el principio de la autonomía universitaria y limitaba la participación estudiantil en los órganos de gobierno de las universidades. Esta ley establecía que el Rector de la facultad sería designado por el Poder Ejecutivo. Este gesto era para los intelectuales, una amenaza directa a la autonomía universitaria y con ella a los ideales de la Reforma de 1918. Esta situación ponía de manifiesto una concepción no liberal de la educación por parte del gobierno de Juan Domingo Perón, concepción que se encontraba en una línea opuesta a la de un gran número de intelectuales. Esta inclinación hacia el liberalismo generó que muchos de ellos se nuclearan en el antiperonismo. Es interesante mencionar que en estos sectores sucedía lo contrario que en el resto de la sociedad: mientras que en esta última la mayoría eran peronistas, en los sectores intelectuales sólo una minoría simpatizaba con el gobierno ${ }^{4}$.

Esto contribuyó a generar que, durante los años del gobierno peronista, prolifere la vida intelectual fuera de las instituciones oficiales. El Colegio Libre de Estudios Superiores y el Instituto Libre de Segunda Enseñanza, funcionaron como centros de estudio alternativos en los cuales se dictaban cursos de distintas asignaturas. Asimismo, durante estos años, prolifero la producción de revistas culturales, literarias y artísticas. Estas revistas y las instituciones antes mencionadas conformaron espacios de producción y difusión de trabajos así como también instancias de legitimación y de sociabilidad fuera de las instituciones oficiales. Dentro de las publicaciones periódicas alternativas podemos ubicar entonces a la revista Imago Mundi que es la que nos interesa abordar.

Para pensar respecto de la noción de intelectuales y cuál es el tipo de representación que hay del rol detrás de su praxis, tomaremos como referencia la noción de "redes intelectuales" de Eduardo Devés Valdés. En principio este autor indica que la noción de "intelectual" tiene que ver con quienes "ejercen la investigación y la docencia a nivel superior, incluyéndose también en numerosas oportunidades a escritores, políticos, diplomáticos, profesionales liberales y líderes sociales que, por su trabajo, son reconocidos como pares al interior del campo"5. Sobre la base de esta noción, entiende que las "redes intelectuales" son "un conjunto de personas ocupadas en la

\footnotetext{
${ }^{3}$ Cf. Pablo Buchbinder, Historia de la Facultad de Filosofía y Letras de la Universidad de Buenos Aires, EUDEBA, Buenos Aires, 1997, 182-183.

${ }^{4}$ Oscar Terán, Historia de las ideas en la Argentina. Diez lecciones iniciales, 1810-1980, Siglo XXI Editores, Buenos Aires, 2008, 257.

${ }^{5}$ Eduardo Devés Valdés, Hacia la constitución de una comunidad intelectual, Colección Idea, Universidad de Santiago de Chile, 2007, 30.
} 
producción y difusión del conocimiento, que se comunican en razón de su actividad profesional, a lo largo de los años"6. Debido a que nos interesa justamente el vínculo de los intelectuales y es por ello que nos interesa pensar a partir de la noción de "redes intelectuales" es que tomaremos lo que señala el autor respecto de la comunicación de los intelectuales entre sí: "Los encuentros cara a cara, la correspondencia a través de diversos soportes y los contactos telefónicos dan lugar a congresos, campañas, publicaciones, comentarios o reseñas de libros, citaciones recíprocas y otras tantas formas en que se establecen articulaciones en el mundo intelectual. No es menos cierto que estas mismas dan origen o se superponen con otros tipos de relaciones: afectivas, familiares, políticas o religiosas, etc."7.

Dado que esta noción incorpora otras dimensiones además de la propiamente laboral, nos permite pensar en la estrecha relación que encontramos entre política y cultura durante este periodo. Ya que, en muchos casos, las diferencias políticas con el gobierno de Perón llevó a ciertos intelectuales a rechazar de plano también el proyecto cultural de este gobierno. En este sentido, la idea de redes nos permitirá comprender las relaciones de estos intelectuales más allá de las instituciones a las que pertenecen, permitiéndonos incorporar en el análisis la dimensión de la sociabilidad.

En este contexto también es importante mencionar que desde 1910 hasta este periodo, el mercado de las revistas en general fue en aumento y esto permitió que adquirieran características especiales en función de un público cada vez más específico. En el caso particular de las revistas culturales, como es el caso de Imago Mundi, pueden caracterizarse como espacios de sociabilidad, legitimación y consagración paralelos o independientes de las instituciones académicas ${ }^{8}$. Alejandro Eujanian también menciona que en las revistas literarias lectores y escritores pertenecen a un mismo ámbito y que, además compiten por el reconocimiento en ese mismo ámbito. Es por ello que, para este autor, las revistas se constituyen en un espacio que permite la conformación de un grupo y la difusión de sus ideas y de sus obras. Asimismo, muchas veces se inscriben en una línea antagónica o al menos paralela al mundo académico y al Estado instalando un espacio diferente de legitimación de la labor intelectual. Por ello, quienes participan en ellas con sus colaboraciones no buscan necesariamente un rédito económico, sino más bien simbólico: buscan instalarse en ese ámbito intelectual. En este sentido también estas revistas ofrecen a sus lectores una biblioteca que ellos mismos forman a partir de ciertas cuestiones estéticas o ideológicas.

Tomaremos también las consideraciones sobre las revistas que hace Beatriz Sarlo. Según esta autora hay dos ideas que suelen acompañar siempre el surgimiento de una revista cultural: la necesidad y el vacío. Esto quiere decir que aparece una necesidad de comunicar algo pero no hay un lugar desde el cual hacerlo. Esto

\footnotetext{
6Ibídem.

${ }^{7}$ Ibídem. La cursiva es nuestra.

8 CF. Alejandro Eujanian, Historia de revistas argentinas (1900-1950). La conquista del público, Asociación Argentina de Escritores de Revistas, Buenos Aires, 1999, págs. 166-167
} 
implica, desde ya, un claro impulso hacia el ámbito público que tiene que ver con el presente. Esto quiere decir que la temporalidad que suponen las revistas culturales es la del puro presente, a diferencia de los libros, que tienen una proyección a mediano o a largo plazo. De este modo la temporalidad de las revistas, permite realizar intervenciones que exige la coyuntura en un determinado momento. Esto implica también cierta necesidad de alineamiento, cierto compromiso, ya que la intervención en la coyuntura supone hacerlo siempre desde una toma de posición. Es por ello que en las revistas hay una fuerte politización que va desde los problemas que eligen poner en su centro hasta su orden y paginación. En este sentido, los debates siempre se llevan adelante en las páginas de las revistas. Otra de las características que menciona Sarlo y que será importante en el trabajo es la de la pluralidad: las revistas culturales suelen ser la representación de un colectivo que la impulsa. Asimismo, otro punto importante que señala Sarlo y que creemos que es relevante para el análisis tiene que ver con que las revistas tienen una doble geografía: aquellos espacios por los que efectivamente la revista circula y aquellos espacios por los que sus integrantes imaginan que circula.

Sarlo también se refiere a las políticas de traducción que las revistas sostienen y señala algo que es relevante para el caso que analizaremos aquí. Es en función de ésta política que Sarlo afirma que podemos leer cómo un colectivo intelectual piensa su intervención en el ámbito público y su inclusión en determinada tradición cultural. Así también afirma que la mayoría de las revistas de la época que nos interesa analizar tienen un programa para modificar el canon. Puntualmente, a la hora de referirse a las revistas de este período, Sarlo menciona el problema de la relación con la cultura europea. Hay en esta época un debate respecto de europeísmo sí o europeísmo no. La autora sostiene que todas las revistas latinoamericanas parten de cierta conciencia de participar de un campo cultural periférico.

En este contexto que mencionamos y en el que aparece la revista Imago Mundi, veremos cómo las caracterizaciones de Sarlo y de Eujanian nos sirven para pensar este caso puntual.

\section{Imago Mundi: Revista de historia de la cultura}

La revista Imago Mundi aparece por primera vez en 1953 y publicará doce números hasta 1956. El primer número aparece gracias al apoyo económico de Alberto Grimoldi, empresario del calzado, quien aportó financiamiento para el primer número de la revista y una oficina ${ }^{9}$. Asimismo, la revista tenía muchos avisos y suscriptores que permitieron la continuidad de la revista aun luego de que el dinero

\footnotetext{
${ }^{9}$ Cf. Omar Acha, "Imago Mundi (1953-1956) en una coyuntura historiográfico política" en Prismas, Revista de historia intelectual, N3, 1999, pág. 120.
} 
de la inversión inicial se terminara ${ }^{10}$. El consejo de redacción fue integrado por: Luis Aznar, José Babini, Ernesto Epstein, Vicente Fatone, Roberto Giusti, Alfredo Orgaz, Francisco Romero, Jorge Romero Brest, José Rovira Armengol, Alberto Salas, Juan Mantovani y León Dujovne. La secretaría de redacción del número 1 al 9 la ocupó Ramón Alcalde y desde el 10 al 11-12 Tulio Halperín Donghi. Muchos de los que participaron de la revista son ex docentes de universidades nacionales y estudiantes, en las palabras del propio José Luis Romero "la revista se transformó en un centro de unión de muchos profesores que habían salido de la Universidad en el 46"11. Asimismo, Tulio Halperin Donghi menciona en Son memorias su vínculo con Romero "Fue el ejemplo de José Luis Romero, quien en esa coyuntura me proporcionó el role model que en el país donde vivo es considerado indispensable para definir con éxito la vocación y luego perseverar en ella"12. No es sólo el caso de Romero, sino también casos como los del José Babini o Vicente Fatone, ya formaban parte del ámbito académico del periodo. En este sentido, los intelectuales que habían quedado fuera de la universidad se constituyeron en referentes para quienes estaban dentro de ella. Los vínculos personales, anteriores a la aparición de Imago Mundi, permitirán ir conformando el grupo. Ramón Alcalde, por ejemplo, tenía una estrecha vinculación con los hermanos Viñas y son ellos quienes lo ponen en contacto con José Luis Romero quien buscaba un secretario para la revista13.

Al comienzo la revista cuenta con una sección de avisos y publicidad: algunas de productos comerciales y otras de revistas contemporáneas como Sur o Ver y Estimar. Luego algunas secciones que se repiten número a número: "Ensayos", "Notas", "Textos y documentos para la historia de la cultura", "Crónica" (donde aparecen crónicas de Congresos), "Reseñas” y "Bibliografía para la historia de la cultura”. En estas últimas tres secciones los textos son breves y con una tipografía más pequeña que la que se usa en las dos primeras. En la sección "Crónica” encontramos una gran cantidad de descripciones de congresos, simposios y jornadas internacionales. Este gesto también se ve en la sección dedicada a reseñar y a comentar bibliografía extranjera. De este modo, la universidad que ha quedado excluida de la universidad oficial muestra en las páginas de Imago Mundi ciertas lecturas que también han quedado fuera de los escenarios oficiales y que consideran fundamentales.

El subtítulo de la publicación "Revista de historia de la cultura" requiere una aclaración de qué es lo que se entiende por "cultura”: “[...] el conjunto de todos los productos de la actividad espiritual del hombre en cuanto ponen de manifiesto esta actividad"14. Indican asimismo que el contenido de la revista o mejor dicho, los intereses de quienes participan allí estarán orientados a "la historia política, la

\footnotetext{
10 Cf. Félix Luna, Conversaciones con José Luis Romero sobre una Argentina con historia, política y democracia, Editorial de Belgrano, págs. 154-155.

11 Ibíd. Pág. 154.

12 Tulio Halperin Donghi, Son memorias, Siglo XXI, Buenos Aires, 2008, pág. 182. La cursiva está en el original.

13 Cf. Ibíd. Pág. 282

${ }^{14}$ Imago Mundi, "Presentación”. Imago Mundi. Revista de historia de la cultura, Número 1, año 1, Buenos Aires, junio de 1953, pág. 1
} 
historia de las ideas en general y la historia de las diversas formas de saber y de creación: filosofía, música, literatura, derecho, ciencias, educación, artes plásticas, etc."15. Incorpora también el criterio de unión, de coincidencia entre quienes participan de la revista: "Imago Mundi quiere ser un territorio de coincidencia"16. Aclara desde el principio y en relación con el tema de las coincidencias: "Imago Mundi destacará una peculiar dimensión de la existencia, porque quienes se agrupan a su alrededor la perciben intensamente y estiman que es decisiva. Sin duda quedarán fuera ciertos temas y aspectos de la cultura que corresponde tratar a otros"17. Esta idea del territorio de coincidencia, también es mencionada por José Luis Romero en el artículo de este número 1 "Reflexiones sobre la historia de la cultura". Artículo en el cual definirá en qué consiste esta disciplina, su objeto, su método y hasta su relación con la filosofía del siglo XIX. Así, haciendo hincapié en el aspecto interdisciplinario de la historia de la cultura, Romero afirma que esta disciplina es "un territorio de coincidencia hacia el que convergen las investigaciones de cada plano de la vida histórica"18. Dará un paso más al afirmar que este territorio de coincidencia "es la vida histórica misma"19. El modo de entender la historia que se propone desde las páginas de la revista, lo aleja de la concepción de la historia política. Marca así su diferencia con quienes integraban la Nueva Escuela Histórica, mayoritaria en los claustros universitarios durante el peronismo, quienes proponían una historia de tinte político-institucional ${ }^{20}$. El propio José Luis Romero plantea que no compartía la metodología documentalista de sus colegas y que por ello Imago Mundi implicó la defensa de cierto modo de investigar que pretende ir más allá de la mera historia política ${ }^{21}$.

En este sentido, y con la finalidad de trazar este territorio de coincidencias, es interesante mencionar la reseña al Warburg Institute perteneciente a la Universidad de Londres, escrita por el propio José Luis Romero. La reseña que es parte de la sección "Crónica" del número 7 de la revista rescata los métodos de trabajo de este instituto cuyo objetivo es el de rastrear "las supervivencias y afloramientos de la cultura de la antigüedad clásica en la cultura europea"22. La metodología de trabajo de este instituto que se dirige a las totalidades y trabaja desde distintas disciplinas con la finalidad de poder abarcarlas, es rescatada por Romero en el artículo así como también las colecciones y revistas que este instituto produce: Studies of the Warburg Institute, Corpus Platonicum Medii Aevi, Journal of the Warburg and Courtauld

\footnotetext{
15Ibídem.

16Ibídem.

${ }^{17}$ Ibíd. pág. 2

${ }^{18}$ José Luis Romero, "Reflexiones sobre la historia de la cultura" en Imago Mundi, №1, año 1, junio de 1953, pág. 10.

19 Ibídem.

${ }^{20}$ Buchbinder, Pablo, Historia de la Facultad de Filosofía y Letras de la Universidad de Buenos Aires, EUDEBA, Buenos Aires, 1997, pág. 184.

${ }^{21}$ Cf. Félix Luna, Conversaciones con José Luis Romero sobre una Argentina con historia, política y democracia, Editorial de Belgrano, pág. 153.

${ }^{22}$ José Luis Romero "El Warburg Institute de la universidad de Londres", Imago Mundi. Revista de la historia de la cultura, Buenos Aires, Número 7, Año II, marzo de 1955, pág. 71
} 
Institute y Mediaeval and Renaissance studies, todos ellos dedicados al estudio de la tradición clásica europea. Podemos pensar este instituto como un modelo para este grupo de intelectuales ya que encontramos ciertos aspectos similares en el modo de trabajo tal como son expresados en el primer editorial de Imago Mundi en el número 1. Allí también se pone de manifiesto un método que tiene que es propio de la historia de la cultura y tiene que ver con seleccionar "los hechos en cuanto encierra una significación para el complejo cultural del cual son efectos remotos"23. En este sentido, la búsqueda de interlocutores fuera de la Argentina no tiene solo que ver con un diagnóstico de vacío de proyecto cultural, sino con una visión de la historia que está en una línea distinta del modo en el cual se piensa y se hace historia en nuestro país. De este modo, la cuestión también es metodológica. Esto se ve en la reseña que escribe Javier Malagón en el №1 de la revista. Allí habla sobre el programa de historia de América como un proyecto en el cual trabaja una comisión de Historia de Instituto Panamericano de Geografía e Historia con el financiamiento de la Fundación Rockefeller. Este programa que comenzara a trabajar en La Habana y luego se reunirán en México, apunta al estudio de "América en su unidad y variedad y como parte de la historia del mundo" 24 .

Esta preeminencia de lo que se produce en el exterior también aparece en reseñas. En esta sección nos encontraremos en su mayoría con textos escritos en Europa. El lugar desde el que se enuncia en Imago Mundi tiene que ver con reconocer una familiaridad o un interés con lo que se está produciendo fuera. En el número 5 de la revista nos encontramos con una reseña de la revista Encouter, revista que aparece en Londres. La dirección es compartida por intelectuales de renombre como Karl Jaspers y reciben colaboraciones de intelectuales de todas las nacionalidades. En el mismo número aparece una reseña de The times literary supplement, particularmente a un número aparecido en 1953. También se hace referencia a un Congreso de Sociología realizado en México en ese año y a un Congreso de Psicología realizado en el mismo país. Así como también un Congreso Internacional de Filosofía realizado en San Pablo en el mismo año. Si volvemos a lo planteado por Beatriz Sarlo respecto de que las revistas implican una intervención en el presente e implican asimismo un alineamiento ideológico-político, vemos cuál es la intención de este grupo de intelectuales. Sus intereses los ubican más allá de lo que está sucediendo en el país y hacia allí se dirigen, generando un espacio de consulta que es alternativo a lo que está circulando en la universidad de esos años. Hay allí también una propuesta alternativa al canon institucional de esos años. Todo esto nos permite pensar que las redes que están intentando trazar este grupo de intelectuales es desde Argentina hacia el mundo, fundamentalmente hacia Europa.

\footnotetext{
23 Imago Mundi, "Presentación”. Imago Mundi. Revista de historia de la cultura, Número 1, año 1, Buenos Aires, junio de 1953, pág. 2

${ }^{24}$ Javier Malagón, "Programa de historia de América”, Imago Mundi. Revista de historia de la cultura, Buenos Aires, Número 1, Año I, junio de 1953, pág. 125
} 
Estos vínculos que hemos mencionado, estas redes que desde la revista se trazan con Europa y otros países importantes del mundo, tienen también su reciprocidad. Así lo comenta José Luis Romero cuando, en ocasión de la visita del filósofo italiano Michele Federico Sciacca a Buenos Aires, se hizo un coloquio al que fue invitada lo que Romero llama "universidad oficial" así como también se invitó a los intelectuales nucleados en Imago Mundi. Otra intervención en la revista que muestra los vínculos de estos intelectuales con aquellos interlocutores en el exterior es la crónica "Museo del hombre en Paris" escrita por Paul Rivet para ser publicada en Imago Mundi. Asimismo, se ve también en la crónica "Relaciones culturales entre el viejo y el nuevo mundo" escrita por Juan Mantovani y publicada en el №4 de la revista. Allí Mantovani reseña una actividad de la UNESCO que pretende estudiar las relaciones entre América y Europa. Encuentra como antecedente de esta actividad las reuniones "Entretien" que organizo en Instituto Internacional de Cooperación Intelectual a iniciativa del PEN Club de la Argentina y que se realizó en 1936 cuyo tema fue Europa-América Latina. En este sentido, también se acentúa el vínculo que puede establecerse entre lo que se está pensando fuera del país y lo que se está pensando dentro. También menciona esto en sus memorias Tulio Halperin Donghi, "Esa inserción en el circuito internacional de la disciplina, hecha posible con el prestigio en el él había ganado ya Romero, no habría con todo podido materializarse tan rápidamente si no hubiera tenido a su servicio toda la tenacidad que el secretario de Imago, Ramón Alcalde, ponía en sus esfuerzos por establecer acuerdos de intercambio con periódicos de ambos mundos"25.

En el último número publicado en 1956 -un número doble-, aparece un cambio en el contenido de la revista. En lugar de publicar diferentes textos de distintos temas, abren el número con una pregunta: “Existe una crisis contemporánea?”. Respecto de esta pregunta, se pronuncian distintas personalidades de la intelectualidad internacional como Walter Goetz -historiador-, Eduard Spranger y Jean Wahl ambos filósofos. Y dentro de los intelectuales nacionales: Francisco Romero, Francisco Ayala, Sebastián Soler, Gino Germani, Renato Treves, José Babini, Guillermo de Torre, Tulio Halperín Donghi, Juan Mantovani, Gustavo Beyhaut, Jaime Rest, P. O. Dudgeon, León Rozitchner y Marcos Victoria. Muchos de ellos, miembros activos de la revista. En principio aparece una presentación de la problemática que funciona como eje del número. Luego aparecen los diagnósticos de distintas personalidades de la intelectualidad internacional y nacional.

En el artículo que escribe Tulio Halperín Donghi "Crisis de la historiografía y crisis de la cultura", deja ver que el problema que atraviesa la disciplina tiene que ver con una falta de acuerdo entre los intelectuales sobre los temas y los métodos de trabajo. Incluso la crisis alcanza el acuerdo sobre la propia disciplina: "los historiadores no están ya de acuerdo sobre qué sea la historia"26. Para este autor la crisis de la historiografía es asimismo la crisis de Occidente: es una crisis social y cultural.

\footnotetext{
25 Tulio Halperin Donghi, Son memorias, Siglo XXI, Buenos Aires, 2008, pág. 281.

${ }^{26}$ Tulio Halperin Donghi, "Crisis de la historiografía y crisis de la cultura". Imago Mundi, Número 1112, Año III, marzo-junio 1956, pág. 96.
} 
También Francisco Romero escribe sobre el tema y hace un diagnóstico de la crisis. Así como Halperín Donghi, Francisco Romero opina que la crisis tiene que ver con una falta de visión compartida del mundo. Para este autor la crisis es profunda a tal punto que pone en cuestión los pilares más importantes de occidente: individualismo, intelectualismo y activismo ${ }^{27}$. Son los pilares de la cultura los que se tambalean. Romero en su diagnóstico de la crisis menciona: "El planeta se ha unificado por la celeridad de los medios de comunicación y transporte, la interdependencia traída por el nivel internacional de la industria y el comercio, y la acción unificante de las dos grandes guerras; todo él vibra ahora al unísono, lo ocurrido en cada parte repercute al punto en las demás y todo problema de importancia asume el relieve de problema mundial" ${ }^{28}$. En líneas generales los artículos de este número de la revista giran en torno de este tema: la crisis contemporánea y sus consecuencias. Pensamos que en este sentido, al leer el último número que sale en 1956, puede hacerse un diagnóstico retrospectivo. Es decir que a la luz de lo que se plantea en este último número: el diagnóstico de una crisis de la historia como disciplina a la vez que de una crisis social y cultural, se puede dotar de sentido a la publicación. Este último número plantea concretamente el terreno de coincidencias que se advertía desde el primer número. Un grupo de intelectuales que ven su disciplina en crisis y que en función de ello, plantean una revista para pensar esa temática.

No está de más señalar que muchos de los que participan de la revista, pasarán a tener importantes cargos en la universidad luego de la caída del gobierno de Juan Domingo Perón. José Luis Romero por caso, será el rector de la facultad. Esto ocasionará la interrupción en la aparición de la revista. Es por ello que Terán llama a este grupo "la universidad del relevo", ya que pasarán a formar las filas de quienes serán figuras reconocidas de la intelectualidad porteña hasta 1966.

Tal como señala Omar Acha y como hemos mencionado más arriba, el antiperonismo permitirá colaboraciones entre distintas publicaciones de la época ${ }^{29}$. Esto tiene que ver con la noción de "redes intelectuales". En este sentido la reseña a Imago Mundi que hace $\mathrm{Centro}^{30}$ lo que hace es trazar entre estas publicaciones una red. Red que supone también una afinidad ideológica y de visión de la coyuntura. Asimismo en Centro se reseñará otra publicación contemporánea antiperonista: la revista Contorno (1953-1959). No está de más mencionar que los hermanos Viñas participarán activamente en ambas publicaciones. Asimismo Tulio Halperín Donghi participará en las tres publicaciones. Podríamos ver aquí cierto gesto similar al que veremos luego en Centro. Frente a cierto diagnóstico de aislamiento cultural, la publicación asumirá la tarea de vincularse con lo que se está produciendo fuera de

\footnotetext{
${ }^{27}$ Francisco Romero, "Diagnóstico y pronóstico de la crisis". Imago Mundi. Revista de la historia de la cultura, Buenos Aires, Número 11-12, Año III, marzo-junio 1956, pág. 35.

${ }^{28}$ Ibíd. pág. 36

${ }^{29} \mathrm{Cf}$. Omar Acha, "Revistas de las afueras del peronismo: Contorno e Imago Mundi entre la renovación historiográfica y el proyecto generacional" en El peronismo clásico (1945-1955). Descamisados, gorilas y contreras, Guillermo Korn (Compilador), Paradiso Ediciones, Buenos Aires, 2007.

${ }^{30}$ Nos referimos a la reseña mencionada en la "Introducción" del presente trabajo.
} 
la Argentina. No obstante percibimos una diferencia: mientras que en Centro se hace un diagnóstico explícito de la realidad nacional y se actúa en consecuencia, en Imago Mundi este diagnóstico no aparece explicitado. No obstante, la mencionada cantidad de artículos que refieren a actividades culturales del extranjero, nos permiten inferir cierto diagnóstico en común que genera esta mirada hacia el trabajo intelectual fuera del país. Así como en Centro podíamos trazar una red de sociabilidad intelectual con revistas de Buenos Aires, en el caso de Imago Mundi, podremos hacerlo con revistas que están en el ámbito internacional. El caso de Contorno será también muy diferente tanto al de Centro como al de Imago Mundi, ya que en ella encontraremos claras referencias a la realidad nacional. No obstante, esto no impide las colaboraciones mutuas. Es interesante mencionar la tensión entre estas revistas que comparten colaboradores y una fuerte crítica a lo académico en los términos en los que está planteado en esos años, pero que muestran metodologías distintas para manifestarlo. No obstante, es posible pensar que una cuestión que aparece en las tres publicaciones es la relación entre intelectuales y política. Sin embargo, el modo en que estas publicaciones materializan esta relación es muy diferente. De modo tal que no es necesario esperar hasta 1955 para ver las diferencias dentro del bloque de intelectuales anti-peronistas, sino que estas van delineándose desde estos mismos años.

\section{Algunas consideraciones finales}

El trabajo comienza trazando una red: de Centro a Imago Mundi. Esa red que efectivamente me llevó a las páginas de esta última revista, es la red con la que -en ese momento- los intelectuales de Centro buscan establecer un vínculo de referencia con los intelectuales ya consagrados de Imago Mundi. Con la elaboración de una imagen de poca -o poco interesante- actividad cultural o intelectual en los años peronistas, estos intelectuales conforman espacios de elaboración y difusión de ideas. Algunas cuestiones que hemos mencionado de Imago Mundi nos permiten plantear el interrogante de si esa actividad intelectual era efectivamente nula o si era tal que no valía la pena hacer referencia a ella. En las páginas de Imago Mundi solo encontramos las reseñas de libros de dos autores argentinos: Roberto Giusti y Alfredo Palacios. Nos referimos a las diferencias metodológicas que José Luis Romero tiene respecto de los historiadores de la universidad oficial. En este contexto, los intelectuales de Imago Mundi también escriben tomando referentes, solo que estos no están en la Argentina. La gran cantidad de publicaciones que circulan en esos años por fuera de la universidad oficial es significativa y nos permite pensar en estos proyectos como espacios de búsqueda de legitimidad tal como menciona Eujanian. Legitimidad que ya no encuentran en las academias oficiales.

Cuando desarrollamos el análisis de Beatriz Sarlo, una de las características de las revistas culturales que mencionamos es la de intervenir en el presente. A diferencia de los libros que suponen una apuesta a largo plazo, las revistas tienen un objetivo claro e íntimamente ligado al presente. Intervienen desde un posicionamiento y lo 
hacen para cambiar o al menos cuestionar ese presente. En este sentido, luego de la lectura tanto de Imago Mundi como de otras revistas contemporáneas, si bien es cierto que hay una cuestión de intervención en el presente, es un presente diferente de un diario. En este sentido, la revista parece instalarse en una temporalidad intermedia entre la urgencia del diario y la temporalidad más amplia que instala el libro. Si bien los artículos de Imago Mundi permiten rastrear lecturas contemporáneas con la revista, no parece correr a la velocidad del acontecimiento. No obstante, si encontramos el gesto de incluirse en una tradición cultural. Tal como lo menciona Sarlo, el canon que se va elaborando desde las páginas de Imago Mundi nos permite ver las afinidades tal como mencionamos en el caso de la reseña del Instituto Warburg en Londres. Asimismo, artículos del tipo "La fisonomía de Occidente según Crane Brinton" de Victor Massuh en el número 10 de la revista, o reseñas de libros como Roman der Archeologie, en el número 3 muestran que la mirada de Imago Mundi se está dirigiendo a otros horizontes de producción.

Sin embargo estas redes no sólo se dirigen hacia fuera de la Argentina, sino que dentro del país se trazan vínculos con revistas locales a través de colaboraciones. En algunos casos, los vínculos por compartir espacios de sociabilidad son anteriores a la existencia de la revista y ésta viene a ser un espacio más que estrecha esos vínculos. Las publicaciones en estos años se transformaron en un ámbito en el cual los intelectuales antiperonistas pudieron compartir y producir ideas buscando una legitimidad alternativa a la de la universidad oficial. Sin embargo, esta búsqueda tuvo diferentes características en cada una de ellas. Ya mencionamos cómo Centro y Contorno establecieron otras relaciones con el canon y encontraron otra tradición en la cual inscribirse. En este sentido, ya vemos delinearse desde aquí las diferencias que se materializaran luego de la Revolución Libertadora cuando, sobretodo el núcleo de intelectuales de Imago Mundi acceda a puestos en la universidad.

\section{Bibliografía}

Acha, Omar, "Imago Mundi (1953-1956) en una coyuntura historiográfico política" en Prismas, Revista de historia intelectual, N³, 1999, pp. 117-142.

----------, "Revistas de las afueras del peronismo: Contorno e Imago Mundi entre la renovación historiográfica y el proyecto generacional" en El peronismo clásico (1945-1955). Descamisados, gorilas y contreras, Guillermo Korn (Compilador), Paradiso Ediciones, Buenos Aires, 2007.

Borello, Rodolfo, "Imago Mundi". Centro. Revista del centro de estudiantes de la facultad de Filosofía y Letras, Número 7, Año 3, Buenos Aires, diciembre de 1953.

Buchbinder, Pablo, Historia de la Facultad de Filosofía y Letras de la Universidad de Buenos Aires, EUDEBA, Buenos Aires, 1997.

Devés-Valdés, Eduardo, Hacia la constitución de una comunidad intelectual, Colección Idea, Universidad de Santiago de Chile, 2007

Dosse, François, La Marcha de las ideas, historia de los intelectuales, historia intelectual, Universitat de Valencia, Valencia, 2007. 
Eujanian, Alejandro, Historia de revistas argentinas 1900-1950. La conquista del público, Asociación Argentina de Editores de Revistas, 1999.

Fiorucci, Flavia, Intelectuales y peronismo (1945-1955), Editorial Biblos, Buenos Aires, 2011.

Haperin Donghi, Tulio. "Crisis de la historiografía y crisis de la cultura". Imago Mundi, Número 11-12, Año III, marzo-junio 1956,

Imago Mundi, “Presentación”. Imago Mundi. Revista de historia de la cultura, Número 1, año 1, Buenos Aires, junio de 1953.

Luna Félix, Conversaciones con José Luis Romero sobre una Argentina con Historia, política y democracia, Editorial de Belgrano, Buenos Aires, 1978.

Malagón, Javier, "Programa de historia de América", Imago Mundi. Revista de historia de la cultura, Buenos Aires, Número 1, Año I, junio de 1953

Ory, Pascal y Sirinelli, Jean François; Los intelectuales en Francia. Del caso Dreyfus a nuestros días, Universitat de Valencia, 2007.

Patiño, Roxana, "Revistas literarias y culturales" en Amícola, José y De Diego, Luis; La teoría literaria hoy: conceptos, enfoques, debates, La Plata, Ediciones Al Margen, Colección "Textos Básicos”, 2008.

Romero, Francisco, "Diagnóstico y pronóstico de la crisis" Imago Mundi. Revista de la historia de la cultura, Buenos Aires, Número 11-12, Año III, marzo-junio 1956.

Romero, José Luis, "Reflexiones sobre la historia de la cultura” Imago Mundi. Revista de historia de la cultura, Buenos Aires, Número 1, Año I, junio de 1953.

"El Warburg Institute de la universidad de Londres", Imago Mundi. Revista de la historia de la cultura, Buenos Aires, Número 7, Año II, marzo de 1955.

Sarlo, Beatriz, "Intelectuales y revistas: razones de una práctica" en América. Cahiers du CRICCAL. Centre de Recherches Interuniversitarire sur les Champs Culturels en Amérique Latine, Presses de la Sorbonne Nouvelle, Université de la SorbonneNouvelle, París III, mayo de 1990.

Sigal, Silvia, Intelectuales y poder en la década del sesenta, Punto Sur Editores, Buenos Aires, 1991.

Terán, Oscar, "De la universidad de las sombras a la universidad del relevo" en Punto de Vista. Revista de cultura, año 11, septiembre-diciembre 1988, Buenos Aires.

-, Nuestros años sesentas. La formación de la nueva izquierda intelectual en la Argentina 1956-1966, Punto Sur Editores, Buenos Aires, 1991.

---------, Historia de las ideas en la Argentina. Diez lecciones iniciales, 1810-1980, Siglo XXI Editores, Buenos Aires, 2008.

Recibido: 06/03/2016

Evaluado: 30/03/2016

Versión Final: 26/04/2016 